\title{
Influence of soil texture on nature of mangrove vegetation in Sundarbans Tiger Reserve forest of India
}

\author{
Dasgupta, $\mathbf{M}^{1}$, A. Ghosh ${ }^{2}$, S. Mukherjee ${ }^{3} \&$ N. Sen Sarkar ${ }^{4}$ \\ ${ }^{1}$ Eutech Scientific Services, New Jersey, USA \\ ${ }^{2}$ Department of Botany, Burdwan University, India \\ ${ }^{3}$ Member Secretary, West Bengal Pollution Control Board, India \\ ${ }^{4}$ Department of Botany, Kalyani University, India
}

\begin{abstract}
Quality of bottom soil forms an important component for any kind of mangrove reforestation as well as coastal rehabilitation program. Since textural composition plays an important role in determining the soil quality, a study was undertaken to assess the influence of variations in soil texture on distribution and abundance of different mangrove species across five zones of Sundarbans Tiger Reserve, India.This study showed these mangrove soils to exhibit good occurrences of sand particles. However,the effects of sands on textural quality were frequently masked by moderate occurrence of finer soil particles which rendered the soils to be largely loamy in texture.Such soil conditions favored establishment of mangrove vegetation. Species wise also, mangrove vegetation was found to be more diverse in loam, clay loam, sandy loam and sandy clay loam soil textures respectively while a very few species were observed in clay, sandy clay and silty clay soils.
\end{abstract}

Keywords - Sundarbans Tiger Reserve forest; soil texture; mangrove vegetation; distribution; abundance.

\section{INTRODUCTION}

Mangrove forests cover an area of approximately 160,000 $\mathrm{km}^{2}$ all over the world, in which the largest forest areas are found in Malaysia, India, Bangladesh, Brazil, Venezuela, Nigeria and Senegal (Giri\&Muhlhausen, 2008; Alongi, 2009). Indian Sundarbans falls under the jurisdiction of North 24-Parganas and South 24-Parganas districts in West Bengal comprising of 19 rural blocks covering a total area of 9630 sq. km. Since 1973, about 2585.10 sq. km area of south-eastern part of Sundarbans mangals were declared as Sundarbans Tiger Reserve. This area of Sundarbans Tiger Reserve includes the land area of $1680 \mathrm{sq}$. $\mathrm{km}$. while the estuarine rivers, creeks and canals cover about $905 \mathrm{sq} . \mathrm{km}$. This entire area under the Sundarbans Tiger Reserve has been further demarcated as "Core Area" (1330.12 sq. km.) and "Buffer Area" (1255 sq. km.). The Indian Sundarbans is bestowed with the highest floral diversity in the form of mangroves, coastal wetland flora, beach flora, marsh and swamp flora (Chanda, 1977; Naskar \& Guhabakshi, 1987; Naskar, 1993; Naskar \&Mondal, 1999). Ghosh et al, 2003 have reported about 110 plant species to occur in this forest of which about 25 species to be true mangroves. Ghosh, et al (2003) also studied on floral diversity of mangroves and mangrove associated species in the Indian Sundarbans.Physico-chemical properties of different mangrove soils in Sundarbans Tiger Reserve have been studied with relation to species diversity(Dasgupta et al 2003).Further studies have shown that mangrove soils vary considerably as compared to the non-mangrove soils even in same locations and also with other mangrove soils under different locations with regard to some properties (Dasgupta et al, 2018).Patterns of tidal inundation also influence soil characteristics that control species zonation of mangrove forest (Banerjee, 1987; Naidoo, 1980; Saha \&Choudhury, 1995). While studying mangrove zonation pattern, several workers have shown that physiological adaptations to such variations may appear to be useful for explaining the observed zonations of mangroves (Smith, 1992, Satyanarayana et al, 2002).

\section{METHOD}

The objective of determining the textural composition of soils is to know the percentage of soil particles contained in different grain size fractions viz. sand, silt and clay and to classify the soils under different textural groups so that the dominant grain sizes present in the soil can be identified easily. The collected soil samples were air dried at a temperature of about $25^{\circ}-30^{\circ} \mathrm{C}$ and relative humidity of about $20 \%-60 \%$ in the laboratory. This was ground with the 
help of wooden mortar and soil texture was determined by International pipette method (USDA,1966).

During the first phase of the study, regular collections of soil samples were done from the Indian part of Sundarbans covering 15 blocks of Sundarbans Tiger Reserve and adjoining areas. These blocks were divided into five major zones viz. (i) Eastern Zone (ii) Western Zone (iii) Central Zone (iv) Northern Zone and (v) Southern Zone according to their occurrence (Table -1). Soil samples, collected extensively from different zones, were processed and analyzed for textural composition by following the procedures mentioned above.

During the second phase of the study, the textural composition of rhizosphere soils under different mangrove vegetations were assessed. Twenty three mangrove species commonly occurring in these soil zones were identified and soil samples were collected from their root zones to assess the preferredtextural nature of soil required for the specific mangroves for their growth and survival.

\section{RESULT AND DISCUSSION}

The textural compositions of the mangrove soils in the Indian Sundarbans Tiger Reserve have been presented in Table-1. As observed from the table, the sand particles occurred in high concentrations in most of these soils. However, the direct effects of sands were frequently countered by moderately good occurrence of comparatively finer particles like clay which rendered these soils to be largely loamy in texture. Since mangrove soils occur in intertidal zones and finer soil particles are generally transported to these zones by tidal water, such accumulation of finer particles in such alluviated soils may be expected. Occurrence of these kinds loamy texture may be helpful to maintain a loose soil condition in mangrove soils encouraging good rooting of mangrove vegetation in such soils. In addition, these soils are likely to provide better plant nutrition to the mangroves than the soils which are coarser in texture. In the present study, only two soil zones viz. Pirkhali in northern zone and Mayadwipin southern zone showed clayey soil texture. Although these two zones are situated in almost opposite situations but they have a similarity that both of them are characterized by dense mangrove vegetation. This might have trapped larger amount of finer soil particles from the tidal water which increased the clay content of the soils. Since variations in textural compositions are associated with differences in nutrient status, chemical and physical properties and also the biological properties of soils (Brady, 1980), such changes in textural compositions are likely to influence the nature of mangrove vegetations under different textural zones.

Textural compositions of rhizosphere soils for different mangrove species commonly found in Sundarbans Tiger Reserve have been presented in Tables 2 to 6 . In the present study, species wise occurrence of different mangrove vegetation appeared to be influenced by the soil textural groups. In Table-7, an effort has been made to identify the mangrove species which were found to be associated with different textural sub-groups of the soils. As observed from the table, loamy textures under different subclasses viz. loam, clay loam, sandy loam and sandy clay loam harbored more diverse occurrence of mangrove vegetation while in clay, sandy clay and silty clay textural groups, only a few species of mangroves survived. Since loamy soils constitute a large share of the variations in textural compositions of mangrove soils of Sundarbans, these soils may be, in general, considered to be conducive for good occurrence of diverse mangrove species.

\section{CONCLUSIONS}

The study showed thatloam and sandy loam soils showed most diverse mangrove species in Indian Sundarbans Tiger Reserve whereas clay, sandy clay and silty clay showed least diversity.Although sand particles often occurred in appreciable concentrations yet the textural composition of these soils were observed to be mostly under different loamy subgroups. This may be due to transportation of finer particles to these estuarine soils through intertidal water.

\section{ACKNOWLEDGEMENT}

The study was carried out under a National Fellow Research Program of Indian Council of Agriculture.

\section{REFERENCES}

[1] Alongi, D., 2009. The Energetics of Mangrove Forests. Springer, New York, USA., ISBN-13: 9781402042713, Pages: 216.

[2] Banerjee, L.K., 1987. Ecological studies on the mangals in the Mahanadi estuarine-delta Orissa: India. Trop. Ecol., 28: 117-125.

[3] Brady, C. Nyle, (1980)Nature and Properties of Soils. Eurasia Publ. New delhi

[4] Chanda, S (1977) An eco-floristic survey of mangroves of Sundarbans, West Bengal, India, Trans. Bose Res. Inst., 40(1): 5-14.

[5] Dasgupta M \& A. Ghosh (2018): A Comparative Study on the Properties of Mangrove and Non - 
Mangrove Soils of Sundarbans, West Bengal, India.

Advances in Clinical Toxicology 2018, 3(2): 000129.

[6] Dasgupta, M, A. Ghosh, S. Mukherjee \& K.R. Naskar (2003): Nature and properties of soils in different parts of Sundarbans Tiger Reserve, India. J. Indian Soc. Coastal agric. Res., 21(2):59-61.

[7] Ghosh, A, S. Mukherjee, N. Sen, M. Dasgupta\& K. R. Naskar (2003). Floral diversity of mangroves and mangrove associated species in the Indian Sundarbans with special reference to distribution and abundance. $J$ Ind. Soc. Coast. agric. Res.21(1):53-58.

[8] Ghosh, A, S. Mukherjee, N. Sen, M. Dasgupta and K. R. Naskar (2003). Checklist of Mangroves and mangroves associated species in Indian Sundarbans. Seshaiyana 10 (2): $211-224$.

[9] Giri, C. and J. Muhlhausen, 2008. Mangrove forest distributions and dynamics in Madagascar (19752005). Sensors, 8: 2104-2117.

[10] Naidoo, G., 1980. Mangrove soils of the Beachwood area, Durban. S. Afr. J. Bot., 46: 293-304.

[11] Naskar, K. R. \& D. N. Guha Bakshi (1987). Mangrove swamps of the Sundarbans - An ecological perspective, Naya Prokash, pp. 1-263.
[12] Naskar, K. R. (1993). Plant wealth of the lower Ganga delta, Daya Publishing House, Delhi. vols. - 1 \& 2, pp. $1-810$.

[13] Naskar, K. R. \& R. N. Mandal (1999). Ecology and Biodiversity of Indian Mangroves. Part I. \& Part -II. 2 Volumes. Daya Publishing House, Delhi - 110 035.pp. $754+163$ Photo Plates.

[14] Saha, S. and A. Choudhury, 1995. Vegetation analysis of restored and natural mangrove forest in Sagar Island, Sundarbans, East Coast of India. Indian J. Mar. Sci., 24: 133-136.

[15] Smith, 1992, Smith, T.J. III. (1992). Forest structure. In "Tropical mangrove ecosystems" (A.I. Robertson and D.M. Alongi, eds), pp. 101-136. American Geophysical Union, Washington DC., USA.

[16] Satyanarayana, B., A.V.Raman, Frank Dehairs, C.Kalavati \& P.Chandramohan (2002) Mangrove floristic and zonation patterns of Coringa,Kakinada Bay, East Coast of India. Wetland Ecology and Management, 20: 25--39

Table.1: Textural compositions of the mangrove soils of Sundarbans Tiger Reserve Forest.

\begin{tabular}{|c|c|c|c|c|}
\hline \multirow{2}{*}{ Zone } & \multirow{2}{*}{ Block } & \multicolumn{3}{|c|}{ Textural composition $(\%)$} \\
\hline & & Sand & Silt & Clay \\
\hline \multirow{3}{*}{$\begin{array}{c}\text { Eastern } \\
\text { Zone }\end{array}$} & Arbesi & $\begin{array}{c}\mathbf{4 9 . 1} \\
41.7-58.5\end{array}$ & $\begin{array}{c}\mathbf{2 8 . 1} \\
25.8-33.5\end{array}$ & $\begin{array}{c}22.6 \\
7.9-31.5\end{array}$ \\
\hline & Khatuajhuri & $\begin{array}{c}\mathbf{4 1 . 6} \\
35.1-48.1\end{array}$ & $\begin{array}{c}\mathbf{2 7 . 1} \\
21.1-33.1\end{array}$ & $\begin{array}{c}\text { 31.2 } \\
18.7-43.7\end{array}$ \\
\hline & Harinbhanga & $\begin{array}{c}\mathbf{4 4 . 3} \\
40.6-48.1\end{array}$ & $\begin{array}{c}\mathbf{2 6 . 8} \\
23.6-30.1\end{array}$ & $\begin{array}{c}\mathbf{2 8 . 7} \\
21.7-35.8\end{array}$ \\
\hline \multirow{3}{*}{$\begin{array}{c}\text { Western } \\
\text { Zone }\end{array}$} & Matla & $\begin{array}{c}\mathbf{4 0 . 7} \\
36.1-46.1\end{array}$ & $\begin{array}{c}\mathbf{2 8 . 1} \\
26.1-32.1\end{array}$ & $\begin{array}{c}\text { 31.0 } \\
21.7-37.8\end{array}$ \\
\hline & Netidhopani & $\begin{array}{c}\mathbf{4 1 . 4} \\
34.8-48.1\end{array}$ & $\begin{array}{c}\mathbf{2 6 . 5} \\
22.9-30.1\end{array}$ & $\begin{array}{c}\text { 31.9 } \\
21.7-42.2\end{array}$ \\
\hline & Chottohardi & $\begin{array}{c}\mathbf{4 2 . 6} \\
36.1-55.7\end{array}$ & $\begin{array}{c}\mathbf{2 9 . 3} \\
26.1-31.0\end{array}$ & $\begin{array}{c}\mathbf{2 2 . 7} \\
14.5-37.7\end{array}$ \\
\hline \multirow{3}{*}{$\begin{array}{c}\text { Northern } \\
\text { Zone }\end{array}$} & Jhilla & $\begin{array}{c}\mathbf{4 9 . 6} \\
15.0-80.6\end{array}$ & $\begin{array}{c}\text { 23.9 } \\
8.2-34.5\end{array}$ & $\begin{array}{c}\text { 32.3 } \\
12.5-75.7\end{array}$ \\
\hline & Pirkhali & $\begin{array}{c}\mathbf{3 6 . 8} \\
25.8-47.8\end{array}$ & $\begin{array}{c}\mathbf{1 0 . 9} \\
0.96-20.9\end{array}$ & $\begin{array}{c}\mathbf{5 2 . 2} \\
51.2-53.2\end{array}$ \\
\hline & Panchmukhani & $\begin{array}{c}\mathbf{5 1 . 8} \\
29.5-60.5\end{array}$ & $\begin{array}{c}\mathbf{3 5 . 3} \\
30.2-49.2\end{array}$ & $\begin{array}{c}\mathbf{1 6 . 1} \\
1.58-22.0\end{array}$ \\
\hline $\begin{array}{c}\text { Central } \\
\text { Zone }\end{array}$ & Chamta & $\begin{array}{c}\mathbf{5 0 . 2} \\
41.6-59.5\end{array}$ & $\begin{array}{c}\text { 30.8 } \\
25.6-36.5\end{array}$ & $\begin{array}{c}\mathbf{1 8 . 8} \\
3.9-32.7\end{array}$ \\
\hline
\end{tabular}




\begin{tabular}{|c|c|c|c|c|}
\hline \multirow{4}{*}{ Chandkhali } & $\mathbf{5 0 . 5}$ & $\mathbf{2 9 . 7}$ & $\mathbf{1 9 . 6}$ \\
& & $38.1-61.0$ & $24.5-33.0$ & $6.0-34.9$ \\
\cline { 2 - 5 } & \multirow{2}{*}{ Goasaba } & $\mathbf{4 2 . 9}$ & $\mathbf{2 6 . 2}$ & $\mathbf{3 1 . 2}$ \\
& & $34.7-55.7$ & $21.7-36.6$ & $4.72-42.5$ \\
\hline \multirow{4}{*}{$\begin{array}{c}\text { Southern } \\
\text { Zone }\end{array}$} & \multirow{2}{*}{ Bagmara } & $\mathbf{3 9 . 9}$ & $\mathbf{2 8 . 1}$ & $\mathbf{3 1 . 8}$ \\
& & $26.2-48.2$ & $23.8-40.2$ & $14.9-40.3$ \\
\cline { 2 - 5 } & \multirow{2}{*}{ Gona } & $\mathbf{4 8 . 2}$ & $\mathbf{2 8 . 3}$ & $\mathbf{2 4 . 0}$ \\
\cline { 2 - 5 } & \multirow{2}{*}{ Mayadwip } & $42.1-55.8$ & $26.0-29.8$ & $14.3-29.0$ \\
\hline & & $\mathbf{3 5 . 0}$ & $\mathbf{2 5 . 0}$ & $\mathbf{3 9 . 9}$ \\
& & $28.1-43.5$ & $21.4-30.5$ & $26.0-48.8$ \\
\hline
\end{tabular}

Table.2: Textural composition (\%) of mangrove rhizosphere soils in eastern zone of Sundarbans Tiger Reserve.

\begin{tabular}{|c|c|c|c|c|c|c|c|c|c|}
\hline \multirow[t]{3}{*}{ Sp. Name } & \multicolumn{9}{|c|}{ Eastern Zone } \\
\hline & \multicolumn{4}{|c|}{ Arbesi } & \multicolumn{2}{|c|}{ Khatuajhuri } & \multicolumn{3}{|c|}{ Harinbhanga } \\
\hline & Sand & Silt & Clay & Sand & Silt & Clay & Sand & Silt & Clay \\
\hline Rhizophora mucronata Lamk & 35.7 & 27.3 & 29.3 & 60.7 & 24.4 & 19.7 & 46.0 & 25.0 & 29.0 \\
\hline Rhizophora. apiculata Blume & 36.8 & 29.1 & 32.2 & 58.7 & 17.3 & 23.1 & 46.0 & 25.0 & 29.0 \\
\hline $\begin{array}{l}\text { Bruguiera gynmorhiza } \\
\text { (L.)Lamk. }\end{array}$ & 56.0 & 8.0 & 36.0 & 47.7 & 21.5 & 30.7 & 45.6 & 23.4 & 31.5 \\
\hline $\begin{array}{l}\text { Bruguiera cylindrica } \\
\text { (L.)Blume }\end{array}$ & - & - & - & - & - & - & - & - & - \\
\hline $\begin{array}{l}\text { Bruguiera sexangula } \\
\text { (L.)Poir }\end{array}$ & 57.0 & 32.0 & 13.3 & 61.5 & 37.5 & 0.98 & 59.6 & 34.2 & 15.7 \\
\hline $\begin{array}{c}\text { Bruguiera parviflora } W . \& \\
A .\end{array}$ & - & - & - & - & - & - & 55.1 & 35.3 & 26.7 \\
\hline Kandelia candel (L.) Druce & 49.5 & 3.98 & 17.0 & 57.5 & 8.0 & 34.5 & 46.1 & 16.1 & 37.7 \\
\hline $\begin{array}{l}\text { Ceriops decandra } \\
\text { (Griff.)Ding. Hou. }\end{array}$ & 54.7 & 27.7 & 17.5 & 43.3 & 34.8 & 19.8 & 48.5 & 32.5 & 18.9 \\
\hline Ceriops tagal (Perr.)Robin & 52.3 & 25.6 & 18.6 & 63.5 & 30.5 & 10.9 & 46.8 & 30.2 & 16.7 \\
\hline Avicennia alba Blume & 54.7 & 27.7 & 22.9 & 52.6 & 28.7 & 12.4 & 44.8 & 27.9 & 14.7 \\
\hline Avicennia officinalis $L$. & 41.7 & 31.5 & 26.7 & 38.5 & 25.6 & 14.6 & 58.5 & 34.5 & 6.9 \\
\hline $\begin{array}{l}\text { Avicennia marina } \\
\text { (Forsk.)Vierh. }\end{array}$ & 51.4 & 32.4 & 17.8 & 50.1 & 33.1 & 16.7 & 54.0 & 31.0 & 15.0 \\
\hline $\begin{array}{c}\text { Sonneratia apetala Buch. } \\
\text { Ham. }\end{array}$ & 49.7 & 27.7 & 0.96 & 56.7 & 30.7 & 12.5 & 41.8 & 29.3 & 18.8 \\
\hline $\begin{array}{c}\text { Sonneratia caseolaris }(L .) \\
\text { Engler }\end{array}$ & - & - & - & - & - & - & - & - & - \\
\hline Sonneratia griffithii Kurz. & 35. & 12.1 & 643.1 & 37.3 & 18.9 & 44.7 & 26.3 & 19.1 & 47.8 \\
\hline Xylocarpus granatum Koen. & 43.1 & 21.4 & 31.2 & 40.1 & 27.1 & 32.7 & 45.9 & 30.9 & 23.0 \\
\hline $\begin{array}{c}\text { Xylocarpus mekongensis } \\
\text { Pierre }\end{array}$ & 54.7 & 27.7 & 17.5 & 56.0 & 11.0 & 9.9 & 55.5 & 34.5 & 33.0 \\
\hline $\begin{array}{c}\text { Aegiceros corniculatum (L.) } \\
\text { Blanco. }\end{array}$ & 41.1 & 24.1 & 34.7 & 54.0 & 8.25 & 37.7 & 45.8 & 31.3 & 22.8 \\
\hline Aegialitis rotundifolia Roxb. & 49.5 & 33.5 & 17.0 & 58.7 & 36.7 & 12.1 & 51.3 & 34.5 & 17.3 \\
\hline Heritiera fomes Buch. Ham. & 48.0 & 23.1 & 28.7 & 48.9 & 21.0 & 29.9 & 56.2 & 12.9 & 30.7 \\
\hline $\begin{array}{c}\text { Nypa fruticans (Thunb.) } \\
\text { Wurmb. }\end{array}$ & 46.8 & 27.3 & 25.8 & 59.9 & 30.8 & 14.2 & 58.7 & 35.6 & 23.4 \\
\hline Phoenix paludosa Roxb. & 51.2 & 37.8 & 10.1 & 49.2 & 29.4 & 8.6 & 60.5 & 34.5 & 5.0 \\
\hline Exoecaria. agallocha $L$. & 49.0 & 29.0 & 21.9 & 47.1 & 29.1 & 23.7 & 52.2 & 31.7 & 16.0 \\
\hline
\end{tabular}

Table.3: Textural composition (\%) of mangrove rhizosphere soils in western zone of Sundarbans Tiger Reserve. 


\begin{tabular}{|c|c|c|c|c|c|c|c|c|c|}
\hline \multirow[t]{3}{*}{ Sp. Name } & \multicolumn{9}{|c|}{ Western Zone } \\
\hline & \multicolumn{3}{|c|}{ Matla } & \multicolumn{3}{|c|}{ Netidhopani } & \multicolumn{3}{|c|}{ Chottohardi } \\
\hline & Sand & Silt & Clay & Sand & Silt & Clay & Sand & Silt & Clay \\
\hline Rhizophora mucronata Lamk & 46.1 & 21.7 & 32.1 & 43.2 & 19.3 & 29.7 & 48.9 & 23.4 & 38.7 \\
\hline Rhizophora. apiculata Blume & 42.4 & 19.7 & 28.0 & 39.7 & 18.1 & 26.4 & 45.6 & 19.5 & 35.7 \\
\hline Bruguiera gynmorhiza (L.)Lamk. & 45.6 & 25.6 & 31.4 & 41.1 & 22.1 & 26.7 & 47.7 & 24.1 & 28.1 \\
\hline Bruguiera cylindrica (L.)Blume & 52.3 & 34.5 & 11.6 & - & - & - & 54.5 & 35.4 & 10.8 \\
\hline Bruguiera sexangula (L.)Poir & - & - & - & - & - & - & 61.2 & 39.8 & 30.2 \\
\hline Bruguiera parviflora $W . \& A$. & 58.7 & 37.8 & 21.2 & 60.6 & 23.1 & 16.1 & 48.2 & 35.2 & 23.5 \\
\hline Kandelia candel (L.) Druce & 47.8 & 24.5 & 31.6 & - & - & - & 51.2 & 26.8 & 37.4 \\
\hline $\begin{array}{c}\text { Ceriops decandra (Griff.)Ding. } \\
\text { Hou. }\end{array}$ & 49.3 & 35.7 & 25.4 & 51.2 & 47.8 & 22.5 & 40.4 & 33.4 & 26.1 \\
\hline Ceriops tagal (Perr.)Robin & 42.1 & 31.6 & 26.1 & 44.3 & 29.7 & 23.1 & 49.5 & 29.2 & 21.1 \\
\hline Avicennia alba Blume & 55.1 & 28.0 & 13.4 & 50.2 & 29.0 & 14.1 & 40.3 & 35.8 & 23.8 \\
\hline Avicennia officinalis $L$. & 43.0 & 25.2 & 30.0 & 35.5 & 25.1 & 24.3 & 48.5 & 34.0 & 26.9 \\
\hline Avicennia marina (Forsk.)Vierh. & 46.5 & 30.2 & 23.2 & 51.2 & 47.8 & 22.5 & 48.2 & 28.2 & 23.5 \\
\hline Sonneratia apetala Buch. Ham. & 53.0 & 42.9 & 24.3 & 51.2 & 47.8 & 22.5 & 42.6 & 33.2 & 24.2 \\
\hline Sonneratia caseolaris (L.) Engler & - & - & - & - & - & - & - & - & - \\
\hline Sonneratia griffithii Kurz. & 35.6 & 29.1 & 39.1 & 31.2 & 26.1 & 38.2 & 37.2 & 23.2 & 39.6 \\
\hline Xylocarpus granatum Koen. & 41.3 & 35.6 & 25.2 & 45.9 & 31.5 & 22.9 & 42.3 & 31.1 & 26.0 \\
\hline Xylocarpus mekongensis Pierre & 41.3 & 35.6 & 25.2 & 46.5 & 31.2 & 27.1 & 42.6 & 33.2 & 24.2 \\
\hline $\begin{array}{c}\text { Aegiceros corniculatum }(L .) \\
\text { Blanco. }\end{array}$ & 40.2 & 23.1 & 33.7 & 51.2 & 47.8 & 22.5 & 39.2 & 25.2 & 35.6 \\
\hline Aegialitis rotundifolia Roxb. & 55.0 & 31.0 & 14.0 & 51.1 & 30.0 & 13.7 & 56.2 & 29.8 & 15.3 \\
\hline Heritiera fomes Buch. Ham. & 44.8 & 19.8 & 35.3 & 43.5 & 16.0 & 31.6 & 45.0 & 18.3 & 34.0 \\
\hline Nypa fruticans (Thunb.) Wurmb. & 57.8 & 22.3 & 20.1 & 59.1 & 21.9 & 18.9 & 56.2 & 21.0 & 17.8 \\
\hline Phoenix paludosa Roxb. & 49.5 & 32.9 & 17.3 & 48.1 & 32.1 & 18.1 & 39.0 & 25.0 & 13.3 \\
\hline Exoecaria. agallocha $L$. & 45.0 & 28.0 & 24.3 & 45.5 & 28.0 & 26.3 & 40.2 & 29.2 & 29.5 \\
\hline
\end{tabular}

Table.4: Textural composition (\%) of mangrove rhizosphere soils in central zone of Sundarbans Tiger Reserve.

\begin{tabular}{|c|c|c|c|c|c|c|c|c|c|}
\hline \multirow{2}{*}{ Sp. Name } & \multicolumn{9}{|c|}{ Central Zone } \\
\cline { 2 - 12 } & \multicolumn{3}{|c|}{ Chamta } & \multicolumn{3}{c|}{ Chandkhali } & \multicolumn{3}{c|}{ Goasaba } \\
\cline { 2 - 12 } & Sand & Silt & Clay & Sand & Silt & Clay & Sand & Silt & Clay \\
\hline Rhizophora mucronata Lamk & 49.6 & 22.2 & 30.6 & 42.1 & 20.1 & 26.3 & 47.1 & 23.7 & 29.1 \\
\hline Rhizophora. apiculata Blume & 29.3 & 23.5 & 27.3 & 31.1 & 25.4 & 29.7 & 33.8 & 27.8 & 31.4 \\
\hline Bruguiera gynmorhiza (L.)Lamk. & 48.5 & 21.5 & 29.8 & 59.1 & 5.7 & 35.1 & 61.2 & 15.4 & 39.8 \\
\hline Bruguiera cylindrica (L.)Blume & 56.7 & 35.7 & 9.8 & 63.4 & 39.7 & 11.8 & 61.0 & 33.0 & 6.0 \\
\hline Bruguiera sexangula (L.)Poir & 46.5 & 27.5 & 25.9 & - & - & - & 59.7 & 34.5 & 25.6 \\
\hline Bruguiera parviflora W. \& A. & 53.2 & 30.2 & 16.5 & 58.7 & 33.7 & 7.5 & 62.1 & 36.9 & 23.4 \\
\hline Kandelia candel (L.) Druce & 54.1 & 28.7 & 34.1 & 59.5 & 33.5 & 36.5 & 56.3 & 28.3 & 37.6 \\
\hline Ceriops decandra (Griff.)Ding. & 49.1 & 33.1 & 17.9 & 54.1 & 32.1 & 15.9 & 50.2 & 36.7 & 29.8 \\
\hline Hou. & & & & & & & & & \\
\hline Ceriops tagal (Perr.)Robin & 60.5 & 34.5 & 4.9 & 49.5 & 30.5 & 7.5 & 58.0 & 30.0 & 4.0 \\
\hline Avicennia alba Blume & 56.5 & 29.5 & 13.9 & 59.0 & 36.7 & 15.7 & 61.0 & 33.0 & 6.0 \\
\hline Avicennia officinalis L. & 53.2 & 30.2 & 16.5 & 55.8 & 32.1 & 17.8 & 61.0 & 33.0 & 6.0 \\
\hline Avicennia marina (Forsk.)Vierh. & 30.2 & 23.2 & 46.5 & 43.8 & 35.2 & 24.1 & 45.0 & 31.0 & 24.0 \\
\hline Sonneratia apetala Buch. Ham. & 53.0 & 42.9 & 24.3 & 50.7 & 46.9 & 23.6 & 42.0 & 33.7 & 26.1 \\
\hline
\end{tabular}




\begin{tabular}{|c|c|c|c|c|c|c|c|c|c|}
\hline Sonneratia caseolaris (L.) Engler & - & - & - & - & - & - & - & - & - \\
\hline Sonneratia griffithii Kurz. & 32.2 & 13.0 & 55.0 & 33.1 & 16.0 & 58.7 & 61.0 & 33.0 & 6.0 \\
\hline Xylocarpus granatum Koen. & 55.9 & 31.2 & 12.9 & 52.3 & 31.5 & 16.0 & 58.9 & 34.5 & 12.0 \\
\hline Xylocarpus mekongensis Pierre & 47.5 & 31.2 & 17.4 & 58.7 & 33.7 & 7.5 & 62.3 & 19.6 & 14.8 \\
\hline $\begin{array}{c}\text { Aegiceros corniculatum (L.) } \\
\text { Blanco. }\end{array}$ & 47.5 & 31.2 & 17.4 & 41.6 & 25.6 & 32.7 & 44.5 & 21.1 & 35.9 \\
\hline Aegialitis rotundifolia Roxb. & 55.0 & 31.0 & 14.0 & 57.6 & 32.0 & 15.1 & 59.1 & 34.2 & 15.6 \\
\hline Heritiera fomes Buch. Ham. & 44.8 & 19.8 & 35.3 & 44.0 & 20.1 & 27.8 & 43.5 & 26.0 & 31.6 \\
\hline Nypa fruticans (Thunb.) Wurmb. & 53.1 & 39.7 & 5.6 & 59.5 & 36.5 & 3.98 & 61.1 & 40.2 & 10.5 \\
\hline Phoenix paludosa Roxb. & 39.5 & 32.9 & 17.5 & 40.1 & 28.8 & 13.4 & 44.5 & 28.9 & 13.0 \\
\hline Exoecaria. agallocha L. & 46.2 & 25.0 & 24.6 & 45.5 & 28.0 & 26.3 & 48.0 & 26.4 & 22.1 \\
\hline
\end{tabular}

Table.5: Textural composition (\%)of mangrove rhizosphere soils in northern zone of Sundarbans Tiger Reserve.

\begin{tabular}{|c|c|c|c|c|c|c|c|c|c|}
\hline \multirow[t]{3}{*}{ Sp. Name } & \multicolumn{9}{|c|}{ Northern Zone } \\
\hline & \multicolumn{3}{|c|}{ Jhilla } & \multicolumn{3}{|c|}{ Pirkhali } & \multicolumn{3}{|c|}{ Panchmukhani } \\
\hline & Sand & Silt & Clay & Sand & Silt & Clay & Sand & Silt & Clay \\
\hline Rhizophora mucronata Lamk & 42.2 & 22.9 & 34.8 & 47.8 & 28.3 & 36.7 & 49.8 & 31.1 & 39.5 \\
\hline Rhizophora. apiculata Blume & 46.0 & 20.7 & 36.7 & 42.2 & 22.9 & 34.8 & 46.2 & 29.1 & 37.7 \\
\hline Bruguiera gynmorhiza (L.)Lamk. & 44.3 & 16.3 & 28.7 & 42.1 & 10.1 & 25.4 & 47.6 & 13.4 & 29.8 \\
\hline Bruguiera cylindrica (L.)Blume & - & - & - & 47.8 & 28.3 & 36.7 & 58.6 & 36.6 & 14.7 \\
\hline Bruguiera sexangula (L.)Poir & 40.3 & 29.7 & 21.8 & - & - & - & 47.8 & 37.8 & 27.6 \\
\hline Bruguiera parviflora $W . \& A$. & - & - & - & 43.2 & 32.1 & 26.7 & 47.1 & 36.6 & 27.9 \\
\hline Kandelia candel (L.) Druce & - & - & - & 45.2 & 21.3 & 32.1 & 49.4 & 23.6 & 33.4 \\
\hline $\begin{array}{c}\text { Ceriops decandra (Griff.)Ding. } \\
\text { Hou. }\end{array}$ & 43.7 & 35.1 & 21.1 & 49.8 & 28.8 & 21.3 & 50.4 & 34.5 & 26.8 \\
\hline Ceriops tagal (Perr.)Robin & 42.3 & 33.4 & 18.7 & 46.7 & 26.7 & 19.8 & 55.0 & 34.4 & 6.8 \\
\hline Avicennia alba Blume & 44.5 & 33.2 & 14.2 & 47.8 & 28.3 & 36.7 & 48.5 & 35.1 & 15.1 \\
\hline Avicennia officinalis $L$. & - & - & - & - & - & - & - & - & - \\
\hline Avicennia marina (Forsk.)Vierh. & 47.4 & 35.6 & 13.1 & 48.5 & 35.2 & 15.2 & 48.0 & 35.0 & 17.0 \\
\hline Sonneratia apetala Buch. Ham. & 45.2 & 25.7 & 12.1 & 46.7 & 30.2 & 13.6 & 41.2 & 28.3 & 18.8 \\
\hline Sonneratia caseolaris (L.) Engler & - & - & - & - & - & - & - & - & - \\
\hline Sonneratia griffithii Kurz. & 32.5 & 19.4 & 44.1 & 34.8 & 22.9 & 42.2 & 33.5 & 20.3 & 46.7 \\
\hline Xylocarpus granatum Koen. & 42.5 & 19.7 & 31.6 & 41.1 & 26.5 & 31.9 & 48.1 & 30.1 & 21.7 \\
\hline Xylocarpus mekongensis Pierre & 44.5 & 25.7 & 17.1 & 46.0 & 20.3 & 13.0 & 45.7 & 24.5 & 13.0 \\
\hline $\begin{array}{c}\text { Aegiceros corniculatum }(L .) \\
\text { Blanco. }\end{array}$ & 44.5 & 21.8 & 37.5 & 47.8 & 28.3 & 36.7 & 45.0 & 25.6 & 36.8 \\
\hline Aegialitis rotundifolia Roxb. & 39.8 & 34.1 & 23.4 & 47.8 & 28.3 & 36.7 & 40.5 & 35.6 & 24.7 \\
\hline Heritiera fomes Buch. Ham. & 45.0 & 21.5 & 33.6 & 43.2 & 22.4 & 35.0 & 46.5 & 25.7 & 31.1 \\
\hline Nypa fruticans (Thunb.) Wurmb. & 51.1 & 36.5 & 13.5 & 50.5 & 33.2 & 14.7 & 49.4 & 32.1 & 14.0 \\
\hline Phoenix paludosa Roxb. & 48.1 & 33.1 & 18.7 & 39.5 & 27.5 & 13.3 & 45.6 & 31.1 & 18.5 \\
\hline Exoecaria. agallocha $L$. & 45.1 & 29.1 & 25.7 & 41.2 & 29.4 & 26.7 & 46.1 & 27.8 & 25.8 \\
\hline
\end{tabular}

Table.6: Textural composition (\%) of mangrove rhizosphere soils in southern zone of Sundarbans Tiger Reserve.

\begin{tabular}{|l|c|c|c|}
\multirow{2}{*}{ Sp. Name } & \multicolumn{3}{|c}{ Southern Zone } \\
\cline { 2 - 4 } & Bagmara & Gona & Mayadwip
\end{tabular}




\begin{tabular}{|c|c|c|c|c|c|c|c|c|c|}
\hline & Sand & Silt & Clay & Sand & Silt & Clay & Sand & Silt & Clay \\
\hline Rhizophora mucronata Lamk & 45.6 & 25.5 & 32.1 & 47.8 & 26.1 & 30.3 & 48.8 & 23.1 & 28.1 \\
\hline Rhizophora. apiculata Blume & 46.7 & 26.6 & 34.5 & 51.1 & 28.9 & 37.1 & 49.7 & 26.9 & 36.2 \\
\hline Bruguiera gynmorhiza (L.)Lamk. & 48.6 & 29.1 & 36.7 & 53.4 & 32.1 & 41.2 & 50.8 & 31.2 & 38.5 \\
\hline Bruguiera cylindrica (L.)Blume & - & - & - & - & - & - & 49.1 & 32.1 & 10.3 \\
\hline Bruguiera sexangula (L.)Poir & - & - & - & - & - & - & - & - & - \\
\hline Bruguiera parviflora W. \& A. & 44.3 & 36.7 & 25.4 & 46.7 & 37.9 & 29.4 & 38.2 & 31.7 & 17.3 \\
\hline Kandelia candel (L.) Druce & 32.7 & 10.5 & 56.7 & 49.1 & 24.7 & 35.6 & 44.4 & 27.4 & 18.2 \\
\hline Ceriops decandra (Griff.)Ding. Hou. & 45.6 & 34.5 & 27.6 & 49.7 & 35.6 & 28.1 & 43.3 & 36.2 & 25.5 \\
\hline Ceriops tagal (Perr.)Robin & 44.1 & 31.2 & 20.6 & 46.7 & 34.5 & 23.5 & 45.1 & 33.4 & 21.4 \\
\hline Avicennia alba Blume & 45.7 & 28.1 & 26.1 & 45.7 & 29.1 & 25.7 & 44.4 & 27.4 & 18.2 \\
\hline Avicennia officinalis L. & 43.2 & 24.2 & 16.5 & 42.1 & 25.0 & 17.0 & 41.0 & 23.0 & 16.0 \\
\hline Avicennia marina (Forsk.)Vierh. & 52.3 & 29.1 & 17.2 & 53.8 & 30.8 & 14.1 & 44.4 & 27.4 & 18.2 \\
\hline Sonneratia apetala Buch. Ham. & 40.1 & 25.0 & 16.2 & 42.3 & 26.2 & 17.1 & 44.4 & 27.4 & 18.2 \\
\hline Sonneratia caseolaris (L.) Engler & 23.7 & 36.5 & 39.7 & - & - & - & - & - & - \\
\hline Sonneratia griffithii Kurz. & 30.7 & 13.6 & 55.7 & 31.1 & 12.0 & 56.1 & 34.5 & 20.0 & 55.3 \\
\hline Xylocarpus granatum Koen. & 43.1 & 25.7 & 35.5 & 44.1 & 26.2 & 37.1 & 45.0 & 26.2 & 35.2 \\
\hline Xylocarpus mekongensis Pierre & 41.5 & 33.0 & 25.1 & 40.4 & 38.7 & 24.3 & 42.6 & 33.2 & 24.2 \\
\hline Aegiceros corniculatum (L.) Blanco. & 53.2 & 23.9 & 48.2 & 39.0 & 24.1 & 35.2 & 41.1 & 24.0 & 38.7 \\
\hline Aegialitis rotundifolia Roxb. & 42.2 & 34.8 & 22.9 & 42.1 & 34.1 & 23.4 & 39.2 & 36.1 & 26.1 \\
\hline Heritiera fomes Buch. Ham. & 44.0 & 22.6 & 35.7 & 44.2 & 22.3 & 35.0 & 53.6 & 24.5 & 30.5 \\
\hline Nypa fruticans (Thunb.) Wurmb. & 50.0 & 35.0 & 14.7 & 50.6 & 36.3 & 14.6 & 49.4 & 32.7 & 16.0 \\
\hline Phoenix paludosa Roxb. & 49.6 & 35.3 & 17.4 & 42.3 & 37.1 & 19.7 & 45.6 & 33.1 & 18.1 \\
\hline Exoecaria. agallocha L. & 44.6 & 29.0 & 26.5 & 43.6 & 27.7 & 26.1 & 45.0 & 27.1 & 25.4 \\
\hline
\end{tabular}

Table.7: Relationship between the soil texture and the different species of mangroves in different zones of Sundarbans Tiger Reserve

\begin{tabular}{|c|c|c|c|c|c|c|}
\hline Clay & Sandy Clay & Silty Clay & Clay Loam & $\begin{array}{l}\text { Sandy Clay } \\
\text { Loam }\end{array}$ & Loam & Sandy Loam \\
\hline \multirow[t]{2}{*}{ S. griffithii } & H. fomes $*$ & S. caseolaris & $\begin{array}{l}\text { R. mucronata } \\
R . \text { apiculata } \\
X . \text { granatum } \\
P . \text { paludosa } \\
\text { E. agallocha }\end{array}$ & \multirow[t]{2}{*}{$\begin{array}{l}\text { B. gymnorrhiza } \\
\text { K. candel } \\
\text { A. officinalis } \\
\text { S. apetala } \\
\text { X. mekongensis } \\
\text { A. corniculatum } \\
\text { H.fomes }\end{array}$} & $\begin{array}{c}\text { B. cylindrica } \\
\text { C. decandra } \\
\text { C. tagal } \\
\text { A. alba } \\
\text { A. marina } \\
\text { X. mekongensis }\end{array}$ & $\begin{array}{c}\text { B. sexangula } \\
\text { B. parviflora } \\
\text { A. rotundifolia } \\
\text { N. fruticans }\end{array}$ \\
\hline & & & $\begin{array}{c}\text { B. } \\
\text { gymnorrhiza* } \\
\text { C. decandra* } \\
\text { A. alba* }\end{array}$ & & $\begin{array}{l}\text { B. sexangula* } \\
\text { B. parviflora* } \\
\text { S. apetala* } \\
\text { A. corniculatum* } \\
\text { A. rotundifolia* } \\
\text { N. fruticans* }\end{array}$ & $\begin{array}{c}\text { B. cylindrica* } \\
\text { C. tagal* } \\
\text { A. alba* } \\
\text { A. marina* } \\
\text { X. granatum* } \\
\text { X. mekongensis* } \\
\text { P. paludosa* } \\
\text { E. agallocha* }\end{array}$ \\
\hline
\end{tabular}

